Research Article

\title{
Characterization and Evaluation of Antibacterial Activity of Silver Nanoparticles Prepared from Sclerocarya birrea Stem Bark and Leaf Extracts
}

\author{
Stephen Nyoni ${ }^{1,2}$, Edson Muzenda ${ }^{2}$, Netai Mukaratirwa-Muchanyereyi ${ }^{2}$ \\ ${ }^{1}$ Chinhoyi University of Technology, Chemistry Department, Chinhoyi, Zimbabwe. \\ ${ }^{2}$ Bindura University of Science Education, Chemistry Department, Bindura, Zimbabwe. \\ Corresponding authors. E-mail: nyonistephen77@gmail.com; snyoni@cut.ac.zw
}

Received: Feb. 9, 2018; Accepted: Sep. 23, 2018; Published: Feb. 27, 2019.

Citation: Stephen Nyoni, Edson Muzenda, and Netai Mukaratirwa-Muchanyereyi, Characterization and Evaluation of Antibacterial Activity of Silver Nanoparticles Prepared from Sclerocarya birrea Stem Bark and Leaf Extracts. Nano Biomed. Eng., 20I9, I I (I): 28-34.

DOI: $10.5101 /$ nbe.v11i1.p28-34.

\begin{abstract}
The synthesis of silver nanoparticles by using the stem extract and leaf extract of Sclerocarya birrea (Marula) and silver nitrate $\left(\mathrm{AgNO}_{3}\right)$ is reported. The synthesized nanoparticles were characterized using ultraviolet-visible spectroscopy, transmission electron microscopy, energy-dispersive X-ray spectroscopy, dynamic light scattering, and visual inspection. The antibacterial activity of the synthesized nanoparticles was done by agar disc diffusion method and tested against Escherichia coli (Gram-negative) and Staphylococcus aureus (Gram-positive). The ultraviolet visible absorption spectrum showed broad absorption band at around $430 \mathrm{~nm}$ corresponding to that of surface plasmon resonance of silver nanoparticles. The synthesis of silver nanoparticles was also confirmed by color change of $\mathrm{AgNO}_{3}$ to reddish brown upon addition of Sclerocarya birrea stem and leaf extracts. In addition, change in color of the solution from colorless to brown within 5 min at $\mathrm{pH} 11$ showed that the rate of reduction was faster at this $\mathrm{pH}$ compared to lower $\mathrm{pH}$. Transmission electron microscopy analysis proved that the synthesized nanoparticles were spherical in shape; energy-dispersive spectroscopy analysis confirmed the presence of silver in the synthesized nanoparticles; and dynamic light scattering confirmed that the stem nanoparticles were generally larger ( 36 to $58 \mathrm{~nm}$ ) than those synthesized from leaf extracts ( 32 to $48 \mathrm{~nm}$ ). These nanoparticles were found to possess potential antibacterial activity against $E$. coli and $S$. aureus.
\end{abstract}

Keywords: Silver nanoparticles; Sclerocarya birrea extracts; Antibacterial activity

\section{Introduction}

Metal nanoparticles (NPs) have received a lot of attention in the recent years due to their optical, electrical and biological properties which make them central to numerous applications such as catalysis, biosensing, imaging, drug delivery, antimicrobial activities and optical spectroscopy [1-4]. Amongst different types of metal nanoparticles, copper, zinc, titanium, magnesium, gold and silver nanoparticles (AgNPs) are of interest because of their potent antimicrobial activity against different types of pathogens such as Bacillus subtilis, Staphylococcus aureus and Klebsiella pneumonia $[5,6]$. Silver has been recognized for producing nanoparticles having inhibitory effect on microbes present in medical and industrial processes $[7,8]$, and the smaller the nanoparticles the greater the antimicrobial effect [9]. This makes synthesis of 
AgNPs using Sclerocarya birrea plant extracts the focus of this current research work.

Current chemical methods of NPs synthesis are suffering from drawbacks like expensive reagent and hazardous reaction conditions; hence the focus towards use of biological synthesis pathways that require less harsh and eco-friendly reaction conditions [10-12]. The use of plant extracts is likely to be a better option for NPs synthesis. Biological synthesis of NPs using plant extracts offers several advantages such as nontoxicity and eco-friendliness. Successful synthesis of NPs using plant extracts has been reported [13-16]. For NPs destined for medical applications, it is imperative to begin with extracts from plants known traditionally to have medicinal properties. In this work, we have chosen Sclerocarya birrea (Marula) plant with special attention to its stem bark and leaves. Sclerocarya birrea plant is a wide spread species throughout the semi-arid deciduous Savannas in Sub-Saharan Africa and locally found in Zimbabwe. The plant contains phytochemicals and reducing sugars [17]. These play a significant role in reduction and stabilization of metal NPs. Mechanisms for synthesis of AgNPs have been proposed [18]. Furthermore, Sclerocarya birrea has been part of civilization since ancient times with use of all parts of the plant for medicinal purposes related to bacterial infections. Bio-synthesis of silver nanoparticles from Sclerocarya birrea stem bark and leaf extracts and their comparative application towards antibacterial activity is lacking in current studies. Individually, silver nanoparticles and Sclerocarya birrea stem barks and leaves have known medicinal properties [17]; hence stabilization of the nanoparticles with phytochemical compounds from stem and leaf extracts is expected to provide a better antimicrobial effect. In the field of nanoscience and nanotechnologies, Sclerocarya birrea has not been used for green synthesis of nanoparticles for nanomedicines despite its traditional medicinal uses.
On top of shortage of medicinal drugs in developing countries, microbes are also becoming more resistant to common drugs used for their treatment. Furthermore, from WHO's list of pathogenic species undergoing resistance to common medicines, $E$. coli falls under the critical priority. It is against this background that the present study aimed at synthesis of silver nanoparticles using the stem and leaf extracts of Sclerocarya birrea, characterization and evaluate their antibacterial activity against E. coli. The dried Sclerocarya birrea stem barks and leaves are shown in Fig. 1(a) and (b), respectively.

\section{Experimental Materials}

Stem barks and leaves of Sclerocarya birrea were obtained from a nearby bushy area at Bindura University of Science Education (BUSE), Deionized water was prepared using Deionizer (Organo F-3 cartridge type water deionizer) at BUSE laboratories, Whatman number 1 filter paper, $\mathrm{HNO}_{3}$, and ethanol were supplied by Sky laboratories, $\mathrm{AgNO}_{3}$ was obtained from Associated chemicals. Gram-positive (Staphylococcus aureus) bacteria and Gram-negative (Escherichia coli) bacteria were obtained from BUSE biological sciences laboratory.

\section{Instruments}

Genesys 10S UV-Vis spectrometer was used for monitoring the bio-reduction of AgNPs by Sclerocarya birrea stem and leaf extracts. Transmission electron microscopy (TEM) images were obtained from a Zeiss Libra TEM 120 Model operated at $90 \mathrm{kV}$. Energy dispersive X-ray spectroscopy (EDS) using INCA Energy TEM200 with analysis software (JOEL) was used for elemental composition analysis on an aluminum mount. A Malvern Zetasizer Nano series

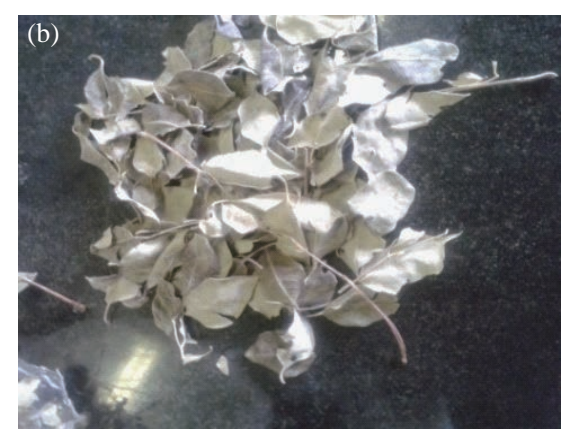

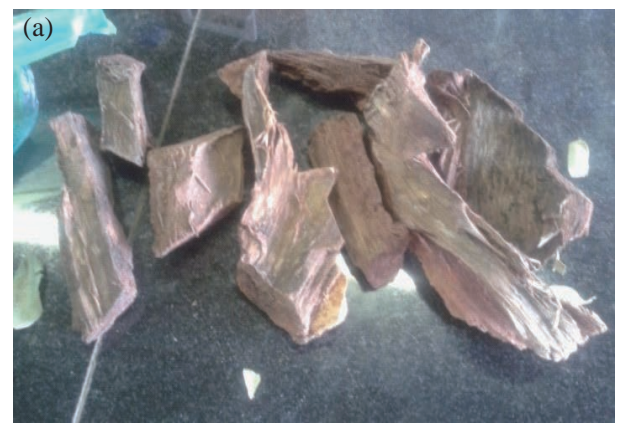

Fig. 1 (a) Sclerocarya birrea dried stems and (b) Sclerocarya birrea dried leaves. 
(dynamic light scattering (DLS)) was used for particle size and particle distribution. Deionizer (Organo F-3 cartridge type water deionizer) was used to obtain deionized water and AD1020 pH/mv/ISE pH meter from Sky labs was used for $\mathrm{pH}$ measurements.

\section{Treatment of glassware}

All glassware were washed with laboratory detergent, rinsed with doubly distilled water then washed in $10 \% \mathrm{HNO}_{3}$ for rinsing purposes. The glassware was then thoroughly rinsed in deionized water to remove impurities and contaminants that may in any case could result in interference.

\section{Preparation of Sclerocarya birrea stem bark and leaf extracts}

Fresh and healthy Sclerocarya birrea stem barks and leaves were washed three times with deionized water to remove dust and impurities. The stem barks and leaves were air dried in the shade at room temperature. The dried samples were ground into fine powder. 15 g of each sample were boiled with $100 \mathrm{~mL}$ deionized water for about $30 \mathrm{~min}$ in an Erlenmeyer flask while continuously stirred. The extract was cooled to room temperature and filtered thrice through Whatman number 1 filter paper. Few drops of ethanol were added as a disinfectant to the filtered samples. The samples were then refrigerated at $4{ }^{\circ} \mathrm{C}$ in a $250 \mathrm{~mL}$ Erlenmeyer flask.

\section{Biosynthesis of silver nanoparticles}

$1 \mathrm{mM}$ solution of silver nitrate aqueous solution was prepared by adding $0.1699 \mathrm{~g}$ silver nitrate (Analytical Grade) to $1000 \mathrm{~mL}$ distilled water. $10 \mathrm{~mL}$ of the Sclerocarya birrea stem and leaf extracts was added to $90 \mathrm{~mL}$ of the prepared $1 \mathrm{mM}$ silver nitrate solution under magnetic stirring. At the end of each reaction time interval, the mixture was collected and subjected to centrifuging at $2000 \mathrm{rpm}$ for $5 \mathrm{~min}$. The supernatant was collected and further subjected to centrifuging at $8000 \mathrm{rpm}$ for $20 \mathrm{~min}$. The supernatant was discarded and the colloidal pellet collected.

\section{Effect of pH on AgNPs synthesis}

$1 \mathrm{M} \mathrm{HCl}$ or $\mathrm{NaOH}$ was added to adjust the $\mathrm{pH}$ of the mixture of plant extracts and silver nitrate solution to pH 4, pH 7, pH 9 and pH 11.

\section{Effect of silver nitrate concentration on AgNPs synthesis}

To investigate the effect of silver nitrate concentration on the formation of silver nanoparticles the following concentrations of silver nitrate were added to $5 \mathrm{~mL}$ of the Sclerocarya birrea stem and leaf extracts (a) $2 \mathrm{mmol}$ (b) $5 \mathrm{mmol}$ (c) $8 \mathrm{mmol}$ (d) 11 mmol (e) $15 \mathrm{mmol}$. These experiments were carried under indoor illumination. The reaction mixtures were sampled for ultraviolet-visible spectroscopy (UV-Vis) analysis (200-800 $\mathrm{nm})$ at time intervals of 1, 24, 48 and $72 \mathrm{~h}$.

\section{Estimation of antibacterial activity}

The comparative antibacterial activities of the plant extracts and the AgNPs synthesized from the respective extracts were effectively accessed against Gram-positive (Staphylococcus aureus) bacteria and Gram-negative (Escherichia coli) bacteria. Disc diffusion method was followed for testing each type of plant extract and their respective AgNPs containing solution. In brief, $25 \mathrm{mg}$ of AgNP pellet were redissolved in $5 \mathrm{~mL}$ distilled water. The discs were separately soaked in plant extracts and solutions containing silver nanoparticles $(5 \mathrm{mg} / \mathrm{mL})$ for $5 \mathrm{~min}$. The discs were air dried in sterile condition. Nutrient agar was used to prepare culture medium. A mass of 23 $\mathrm{g}$ was dissolved in $1000 \mathrm{~mL}$ distilled water. This was heated to boiling to allow complete dissolving of agar powder. A volume of approximately $20 \mathrm{~mL}$ of nutrient agar was poured into glass petri plates and allowed to solidify. The plates containing nutrient agar media were separately streaked with $1 \mathrm{~mL}$ of each microbial culture (used as received) obtained from the Biological sciences department at Bindura University of Science Education. Plates were divided into two and the prepared discs were placed on each part of the plate. The plates were incubated at $37^{\circ} \mathrm{C}$ for $24 \mathrm{~h}$. Maximum zones of inhibition were observed and measured for analysis against each type of test microorganism.

\section{Results and Discussion Visual analysis}

Change of color was the first confirmatory point for the successful synthesis of AgNPs from the plant extracts at different reaction conditions. The change in color is due to the strong absorption of visible light due to excitation of the nanoparticles surface plasmons [19]. The silver nitrate solution turned from clear to a yellowish solution and finally deep brown coloration depending on type of plant extract, reaction time, 
concentration of silver nitrate and $\mathrm{pH}$. The color of the solution in the reaction flask formed an intense brown within 5 minutes for the reaction at pH 11 . The reaction at $\mathrm{pH} 9, \mathrm{pH} 7$ and $\mathrm{pH} 4$ was gradual i.e. the brown color was observed within 30 minutes, 3 hours and 6 hours, respectively. The fast change in the color of the solution at $\mathrm{pH} 11$ indicated that the rate of reduction was faster at this $\mathrm{pH}$ compared to those at lower $\mathrm{pH}$. Basically, the color turned deep brown with rise in $\mathrm{pH}$ from 4 to 11, increase in both reaction time and concentration of silver nitrate solution. This can be ascribed to aggregation due to increase in AgNPs concentration as reaction time, silver nitrate concentration and solution $\mathrm{pH}$ increases. A more intense color was also observed with AgNPs from stem bark extract than leaf extract. Only $2 \mathrm{mmol}$ $\mathrm{AgNO}_{3}$ solution resulted in a yellowish solution and 5 mmol-15 mmol $\mathrm{AgNO}_{3}$ solutions produced an intense coloration at the same reaction time.

\section{Ultraviolet-visible spectroscopy (UV-Vis) analysis}

The surface plasmonic resonance (SPR) peak for the synthesized AgNPs was observed at around $430 \mathrm{~nm}$. This is in agreement with previously reported work [8]. The peak intensities for AgNPs prepared from both the stem bark and leaf extracts increased with increase in sampling time, indicating increase in AgNPs concentration with reaction time. The SPR intensity also increased with increase in pH i.e. from $\mathrm{pH} 4$ to 11 . This was also complemented with the reaction solution becoming deep brown as $\mathrm{pH}$ was adjusted from 4 to 11 . However, the absorption intensity of AgNPs from leaf extract was higher than that of AgNPs from stem (see Table 1). This may suggest that the stem bark extracts have more reducing phytochemical compounds than leaf extracts hence faster reaction for the former than the latter. Furthermore, the absorption intensities increased with increase in $\mathrm{AgNO}_{3}$ concentrations for both stem and leaf extracts.

Table 1 Absorbances of AgNPs synthesized from leaf and stem extracts at maximum wavelength of absorption (430 nm)

\begin{tabular}{ccc}
\hline & \multicolumn{2}{c}{ Absorbance of AgNPs } \\
\cline { 2 - 3 } $\mathrm{pH}$ & Leaf extract AgNPs/430 nm & Stem extract AgNPs/430 nm \\
\hline 4 & 1.01 & 0.46 \\
7 & 1.70 & 0.65 \\
9 & 2.42 & 0.82 \\
11 & 2.55 & 0.84 \\
\hline
\end{tabular}

\section{Energy-dispersive X-ray spectroscopy (EDS) analysis}

Elemental composition of the AgNPs was obtained by EDS analysis, and the spectra are shown in Fig. 2. Two silver peaks were obtained in each case. This can be attributed to the oxidized silver species $\left(\mathrm{Ag}^{+}\right)$and its reduced form (bigger peak). The peak at nearly 3.0 $\mathrm{keV}$ is typical of optical absorption of metallic AgNPs [20]. The $\mathrm{C}$ and $\mathrm{O}$ peaks indicate the presence of some capping organic molecules on AgNPs.

The weight and atomic percent composition of the capped AgNPs are summarized in Table 2.
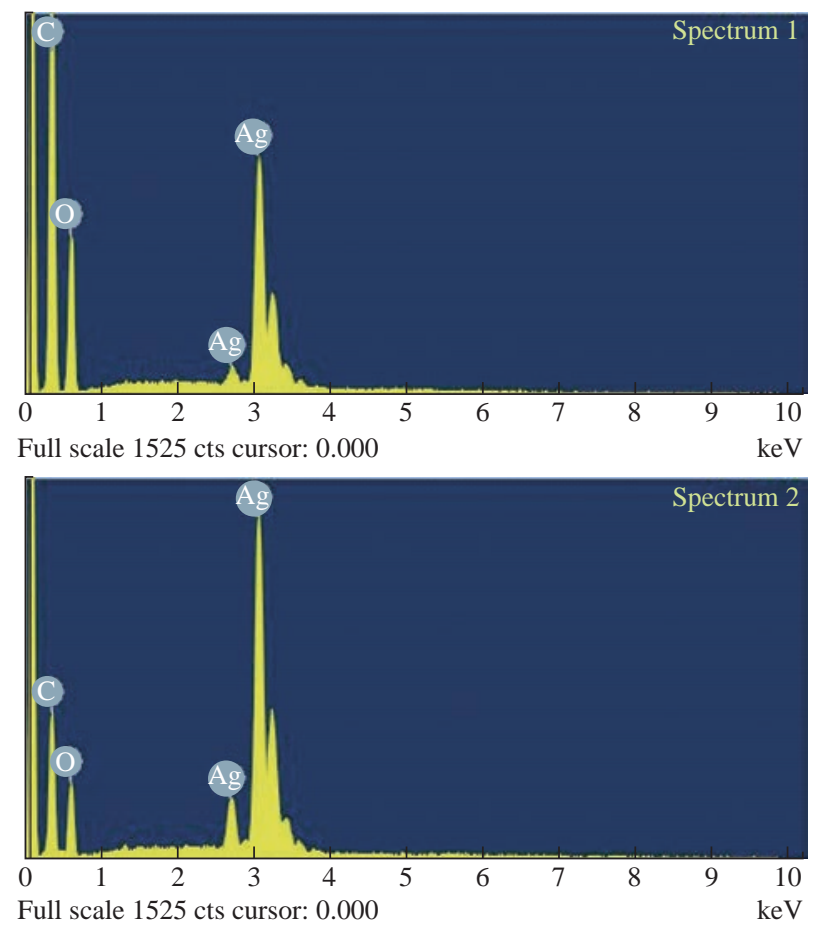

Fig. 2 Energy-dispersive X-ray spectroscopy spectra of AgNPs prepared from $1 \mathrm{mM} \mathrm{AgNO}_{3}$ using leaf extract (spectrum 1) and stem bark extract (spectrum 2).

Table 2 Elemental composition of capped AgNPs from EDS spectral processing

\begin{tabular}{ccc}
\hline Element & Weight (\%) & Atomic (\%) \\
\hline C (K) & 35.80 & 55.24 \\
O (K) & 34.19 & 39.60 \\
Ag (L) & 30.01 & 5.16 \\
\hline
\end{tabular}

\section{Transmission electron microscopy (TEM) analysis}

TEM image of the synthesized AgNPs is shown in Fig. 3. The image shows that the AgNPs are spherical and are polydispersed. This agrees well with broad 


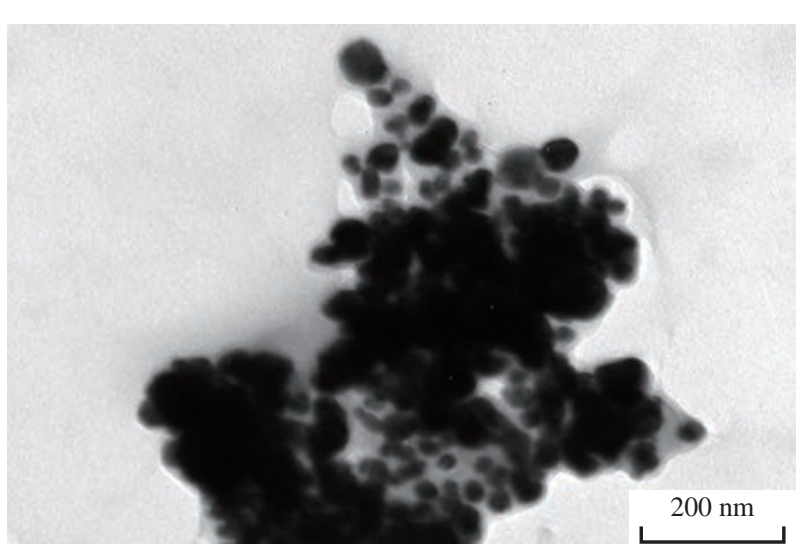

Fig. 3 Transmission electron microscopy image of silver nanoparticles.

spectra observed in UV-Vis analysis.

\section{Dynamic light scattering (DLS) analysis}

Fig. 4 shows particle size distribution of AgNPs prepared from (a) leaf and (b) stem bark extracts. AgNPs synthesized from leaf extract range mainly from 32 to $48 \mathrm{~nm}$, and those from stem bark extract range mainly from 36 to $58 \mathrm{~nm}$. AgNPs from stem bark extract show more polydispersion as indicated by the appearance of two more well defined peaks in the ranges 27 to $35 \mathrm{~nm}, 61 \mathrm{~nm}$ to $67 \mathrm{~nm}$, plus a shoulder peak at around $52 \mathrm{~nm}$.
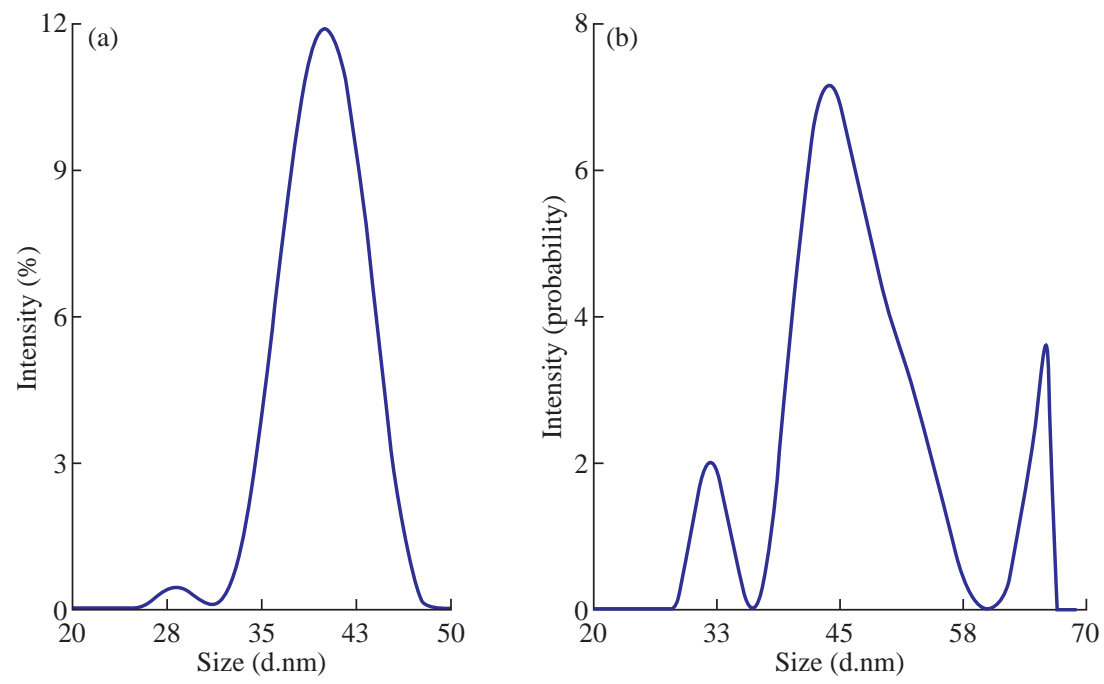

Fig. 4 Dynamic light scattering of (a) leaf extract AgNPS and (b) stem extract AgNPs.

Table 3 Zones of inhibitions found in Staphylococcus aureus and E.coli cultures using plant extracts, AgNPs and Kanamycin (reference drug)

\begin{tabular}{ccccc}
\hline Extracts & Bacteria & By extracts $(\mathrm{mm})$ & By AgNPs $(\mathrm{mm})$ & By Kanamycin $\mathrm{K}_{30}(\mathrm{control})(\mathrm{mm})$ \\
\hline \multirow{2}{*}{ Stem extract } & S. aureus & 2 & 9 & 10 \\
& E.coli & 2 & 3 & 6 \\
\hline \multirow{2}{*}{ Leaf extract } & S. aureus & 1 & 5 & 5 \\
& E.coli & 1 & 2 & 5 \\
\hline
\end{tabular}

\section{Antibacterial activity}

The zone of inhibitions from antibacterial activity of AgNPs, stem extracts, leaf extracts and the reference drug (Kanamycin) are summarized on Table 3.

The results showed that for all materials used, the antibacterial activity was more pronounced against S.aureus in comparison to E. coli. However, AgNPs prepared from stem bark extract had higher antibacterial activity against both bacterial strains used in this work. Furthermore, the results were comparable with the reference drug (Kanamycin); making these AgNPs suitable candidates for treating bacterial infections. The Sclerocarya birrea stem and leaf extracts revealed mild inhibitory activity against both strains i.e. Staphylococcus aureus and $E$. coli. The difference in the zone of inhibition of both bacterial strains could be a result of the cell wall of Gram positive bacteria (S. aureus) which is composed of a thick peptidoglycan layer, consisting of linear polysaccharides chains cross linked by short peptides thus forming more rigid structure leading to difficult penetration of AgNPs compared to the gram negative (E. coli) where the cell wall possesses thinner peptidoglycan layer [21]. Plant-based AgNPs can be used as effective growth inhibitors in various 
microorganisms making them applicable in diverse medical devices and antimicrobial control systems for critical microbes that have developed resistance to current drugs.

\section{Conclusions}

Silver nanoparticles were successfully synthesized using extracts of marula (stem and leaf), a plant found abundantly in Zimbabwe and in Africa at large. As a bio-reducing agent, the results are very promising since the extracts promoted easy formation of nanoparticles at room temperature with fast kinetics and without use of harmful chemicals. The research has managed to prove that Sclerocarya birrea stem and leaf extracts can indeed synthesize silver nanoparticles due to phytochemicals that it contains. The silver nanoparticles synthesized by this method can be strong candidates for use in biological systems. The antibacterial effect of the AgNPs at leaf extract/stem extract was greater than the extracts alone, but further experiments are necessary to determine whether this constitutes a synergic effect. These results have shown that the stem extract is a better reducing agent as compared to leaf extract. Antibacterial activity proved that AgNPs from stem extract inhibited more bacteria multiplication than those from leaf extract. The results of this study proved that the plant biomolecules capped-silver nanoparticles inhibited the growth and multiplication of the bacterial strains such as $S$. aureus and E. coli, making them better candidates for new drug discovery nanotechnologies towards antimicrobial activity.

\section{Acknowledgements}

A special thanks goes to Tebello Nyokong (Distinguished Professor of Medicinal Chemistry and Nanotechnology at Rhodes University, Center for Nanotechnology Innovation) for allowing us to use her nanotechnology equipment to run our samples.

\section{Conflict of Interests}

The authors declare that no competing interest exists.

\section{References}

[1] E.V. Barmina, N.N. Mel’nik, I.I. Rakov, et al., Optical properties of nanocomposites based polymers and metal nanoparticles. Physics of Wave Phenomena, 2017, 25(3): 165-169.

[2] X. Zhao, Y. Xia, Q. Li, et al., Microwave-assited synthesis of silver nanoparticles using sodium alginate and their antimicrobial activity. Colloids and Surfaces A: Physicochemical and Engineering Aspects, 2014, 444: 180-188.

[3] T. Dang-Bao, D. Pla, I. Favier, et al., Bimetallic nanoparticles in alternative solvents for catalytic purposes. Catalysts, 2017, 7(207): 1-33.

[4] C. Raguphathi, J.J. Vijaya, and L.J. Kennedy, Preparation, characterization and catalytic properties of Ni-aluminate nanoparticles: A comparison between conventional and microwave method. Journal of Saudi Chemical Society, 2017, 21: S231-S239.

[5] Y. Zhang, D. Yang, Y. Kong, et al., Synergetic antibacterial effects of silver nanoparticles of aloe vera prepared via a green method. Nano Biomed. Eng., 2012, 2(4): 252-257.

[6] M.A. Bhat, B.K. Nayak, and A. Nanda, Biosynthesis and effect of silver nanoparticles on the efficacy of antibiotics against pathogenic bacteria. Journal of Chemical and Pharmaceutical Sciences, 2014, Special issue 2: 86-89.

[7] R.M. Jose, C.L. Jose, E. Alejandra, et al., The bactericidal effect of silver nanoparticles. Nanotechnol, 2005, 16: 2346-2353.

[8] N.M. Muchanyereyi, T. Muchenje, S. Nyoni, et al., green synthesis of silver nanoparticles using Euphorbia confinalis stem extract, characterization and evaluation of antimicrobial activity. J Nanomater Mol Nanotechnol, 2017, 6: 3 .

[9] C. Baker, A. Pradhan, I. Pakstis, et al., Synthesis and antibacterial properties of silver nanoparticles in natural rubber. Material Chemistry and Physics, 2005, 104: 276283

[10] Y. Rout, S. Behera, A.K. Ojha, et al., Green synthesis of silver nanoparticles using Ocimium sanctum (Tulashi) and study of their antibacterial and antifungal activities. $J$ Microbial and Ant micro, 2012, 4: 103-109.

[11] A. Kedziora, K. Sobik, Synthesis and antibacterial activity of novel titanium dioxide doped with silver. Journal of Sol-Gel Science and Technology, 2013, 62: 79-86.

[12] T. Mochochoko, S.O. Oluwatobi, Green synthesis of silver nanoparticles using cellulose extracted from aquatic weed, water hyacinth. Carbohydrate Polymers, 2012, 98: 290-294.

[13] K. Sahayaraj, S. Rajes, Bio nanoparticles: Synthesis and antimicrobial applications. Science against Microbial Pathogens: Communicating Current Research and Technological Advances, 2011, 1: 228-248.

[14] A.N. Begum, S. Mondal, S. Basau, et al., Biogenic synthesis of $\mathrm{Au}$ and $\mathrm{Ag}$ nanoparticles using aqueous solutions of black tea extracts. Colloids Surfaces B. Bio Interfaces, 2009, 71: 113-118

[15] Q. Huang, Y. Sun, Y. Lu, et al., Biosynthesis of silver and gold nanoparticles by novel sundried Cinnamomumcamphora leaf. Nanotechnol, 2007, 18: 105104.

[16] M.E. Jancy, L. Inbathamizh, Green synthesis and characterization of Nano silver using leaf extract of Morinda pubescens. Asian Journal of Pharmaceutical and Clinical Research, 2012, 5: 159-162.

[17] K.M. Laure, B.V. Penalp, J. Konam, et al., Evaluation of antidiarrheal activity of the fruit-Rind of Picralimanitida (Apolynocea). African Journal of Traditional, Complementary and Alternative Medicines, 2006, 3(4): 66-73.

[18] S. Rajeshkumar, L.V. Bharath, Mechanism of plantmediated synthesis of silver nanoparticles-A review on 
biomolecules involved, characterization and antibacterial activity. Chemico-Biological Interactions, 2017, 273 : 210-227.

[19] J. Devendra, K.D. Hemant, K. Sumita, et al., Synthesis of plant - Mediated silver nanoparticles using papaya fruit extract and evaluation of their anti- microbial activities. Digest Journal of Nanomaterials and Bio structures, 2009, 4(4): 723-727.

[20] K. Jyoti, M. Baunthiyal, and A. Singh, Characterisation of silver nanoparticles using Urtica dioica Linn. Leaves and their synergistic effects with antibiotics. Journal of Radiation Research and Applied Sciences, 2016, 9(3):
217-227.

[21] S. Siddhartha, B. Tanmay, R. Arnab, et al., Synthesis of silver nanoparticles from plant extracts. Nanosci Nanotechnol, 2007, 18: 103-225.

Copyright $₫$ Stephen Nyoni, Edson Muzenda, and Netai Mukaratirwa-Muchanyereyi. This is an open-access article distributed under the terms of the Creative Commons Attribution License, which permits unrestricted use, distribution, and reproduction in any medium, provided the original author and source are credited. 